TRANSACTIONS OF THE

AMERICAN MATHEMATICAL SOCIETY

Volume 355, Number 8 , Pages 3359-3378

S 0002-9947(03)03312-9

Article electronically published on April 25, 2003

\title{
SINGULAR INTEGRALS ON SYMMETRIC SPACES, II
}

\author{
ALEXANDRU D. IONESCU
}

\begin{abstract}
We extend some of our earlier results on boundedness of singular integrals on symmetric spaces of real rank one to arbitrary noncompact symmetric spaces. Our main theorem is a transference principle for operators defined by $\mathbb{K}$-bi-invariant kernels with certain large scale cancellation properties. As an application we prove $L^{p}$ boundedness of operators defined by Fourier multipliers that satisfy singular differential inequalities of the HörmanderMichlin type.
\end{abstract}

\section{INTRODUCTION}

Let $\mathbb{G}$ be a noncompact connected semisimple Lie group with finite center, $\mathbb{K}$ a maximal compact subgroup and $\mathbb{X}=\mathbb{G} / \mathbb{K}$ an associated symmetric space. In this paper we study $L^{p}$ boundedness properties of a class of operators on the symmetric space $\mathbb{X}$ which are the analogue of the singular integral operators on Euclidean spaces. By analogy with the Euclidean case these operators can be defined by Fourier multipliers or by convolution with Calderon-Zygmund kernels.

A necessary condition for $L^{p}$ boundedness of an operator $T_{m}$ defined by a multiplier $m$ is that the multiplier extends to a bounded $W$-invariant holomorphic function in the interior of the tube $\mathcal{T}_{p}=\mathfrak{a}^{*}+i \operatorname{co}\left(W \cdot \rho_{p}\right)$, where $\rho_{p}=|2 / p-1| \rho$. This was observed by Clerc and Stein [5], who also proved a sufficient condition for $L^{p}$ boundedness when the group $\mathbb{G}$ is complex. By analogy with the Euclidean case, a natural theorem is the following: assume that $p \in(1,2) \cup(2, \infty)$ and the multiplier $m$ extends to a holomorphic function in the interior of the tube $\mathcal{T}_{p}$. Assume in addition that $m$ satisfies differential inequalities of the form

$$
\left|\partial_{\lambda}^{j} m(\lambda)\right| \leq A_{j}(1+|\lambda|)^{-j}
$$

for all $\lambda \in \mathcal{T}_{p}$ and any $j \geq 0$, where $\partial_{\lambda}^{j} m(\lambda)$ denotes any partial derivative of $m$ of order $j$. Then the operator $T_{m}$ extends to a bounded operator on $L^{p}(\mathbb{X})$. Statements of this type in various settings can be found in [5], [18, [4], 22], 1], [8] and $[9]$.

In this paper we deal with operators defined by multipliers that are allowed to have singularities on the boundary of the tube $\mathcal{T}_{p}$. These multipliers satisfy differential inequalities of the form

$$
\left|\partial_{\lambda}^{j} m(\lambda)\right| \leq A_{j} d(\lambda)^{-j}
$$

Received by the editors September 12, 2001.

2000 Mathematics Subject Classification. Primary 22E46, 43 A85.

The author was supported in part by the National Science Foundation under NSF Grant No. 0100021 . 
where the function $d(\lambda)$ can vanish on the boundary of the tube $\mathcal{T}_{p}$ (as in (4.1)). These differential inequalities are the analog on symmetric spaces of the classical Hörmander-Michlin differential inequalities. Natural examples are provided by imaginary powers of a suitably modified Laplacian, as in [8]. The associated multipliers have singularities at the points $\left\{w \cdot\left(i \rho_{p}\right): w \in W\right\}$.

The main difficulty in proving $L^{p}$ boundedness of operators defined by multipliers with singularities on the boundary is that the classical Herz majorizing principle [13] fails to apply. As in the case of Euclidean spaces, it is critical to be able to use the large scale cancellation properties of the kernel of such an operator. Our main theorem in this direction is Theorem 3.1-a transference principle that allows one to deal with convolution operators defined by kernels of the form

$$
K(\exp H)=e^{-2 \rho(H) / p} \phi(H),
$$

where $\phi$ is a Calderon-Zygmund kernel on $\mathfrak{a}$. Here $1<p<2$ and the factor $e^{-2 \rho(H) / p}$ is the critical exponential decay factor for $L^{p}$ boundedness. The proof of this theorem is similar to the proof of the corresponding theorem on symmetric spaces of real rank one ([14, Theorem 1]).

Our second main task is to show that this transference principle, together with the Herz majorizing principle and local analysis, suffices to prove $L^{p}$ boundedness of operators defined by multipliers satisfying (4.1). This is significantly harder in the case of general symmetric spaces than in the case of real rank one symmetric spaces. One of the difficulties arises from the fact that the Harish-Chandra expansion of the elementary spherical functions does not converge uniformly in the region close to the walls of the positive Weyl chamber. Thus one cannot get good pointwise estimates on the kernels of the operators in this region. This difficulty was overcome by Anker [1] and we follow his approach. The main observation we need to make is that the volume of this region is smaller than the volume of the full Weyl chamber (in the sense of (4.6)). We will then apply our transference theorem to the part of the kernel supported away from the walls of the positive Weyl chamber. The HarishChandra expansion converges uniformly in this region and the kernel corresponding to the main term in this expansion is of the form (1.2). Also, one can use the Herz majorizing principle to deal with the error terms in the Harish-Chandra expansion in this region.

\section{Preliminaries}

Most of our notation related to semisimple Lie groups and symmetric spaces is standard and can be found, for example, in $[12$. Let $\mathbb{G}$ be a noncompact connected semisimple Lie group with finite center, $\mathfrak{g}$ the Lie algebra of $\mathbb{G}, \theta$ a Cartan involution of $\mathfrak{g}$ and $\mathfrak{g}=\mathfrak{k} \oplus \mathfrak{p}$ the associated Cartan decomposition. Let $\mathbb{K}=$ exp $\mathfrak{k}$ be a maximal compact subgroup of $\mathbb{G}$ and let $\mathbb{X}=\mathbb{G} / \mathbb{K}$ be an associated symmetric space. Let $\mathfrak{a}$ be a maximal abelian subspace of $\mathfrak{p}, \mathbb{M}$ the centralizer of exp $\mathfrak{a}$ in $\mathbb{K}, \Sigma$ the restricted root system of the pair $(\mathfrak{g}, \mathfrak{a})$ and $W$ the associated Weyl group. Let $\mathfrak{a}^{+} \subset \mathfrak{a}$ be a positive Weyl chamber and $\Sigma^{+}$the corresponding set of positive roots. For any root $\alpha \in \Sigma$ let $\mathfrak{g}_{\alpha}$ be the root space associated to $\alpha$ and $m_{\alpha}$ its dimension. Let $\mathfrak{n}=\sum_{\alpha \in \Sigma^{+}} \mathfrak{g}_{\alpha}, \overline{\mathfrak{n}}=\theta(\mathfrak{n}), \mathbb{N}=\exp \mathfrak{n}$ and $\overline{\mathbb{N}}=\exp \overline{\mathfrak{n}}$.

The group $\mathbb{G}$ has an Iwasawa decomposition $\mathbb{G}=\mathbb{K}(\exp \mathfrak{a}) \mathbb{N}$ and a Cartan decomposition $\mathbb{G}=\mathbb{K}\left(\exp \overline{\mathfrak{a}^{+}}\right) \mathbb{K}$. For each $g \in \mathbb{G}$ denote by $H(g) \in \mathfrak{a}$ and $g^{+} \in \overline{\mathfrak{a}^{+}}$ the middle components of $g$ in these decompositions. We will also use the Iwasawa 
decomposition $\mathbb{G}=\overline{\mathbb{N}}(\exp \mathfrak{a}) \mathbb{K}$. We normalize the Haar measure $d k$ on $\mathbb{K}$ with total mass 1 and the Haar measure $d \bar{n}$ on $\overline{\mathbb{N}}$ such that

$$
\int_{\overline{\mathbb{N}}} e^{-2 \rho(H(\bar{n}))} d \bar{n}=1,
$$

where

$$
\rho(H)=\frac{1}{2} \sum_{\alpha \in \Sigma^{+}} m_{\alpha} \alpha(H)
$$

The Haar measure $d g$ on $\mathbb{G}$ can be normalized so that

$$
\int_{\mathbb{G}} F(g) d g=\int_{\overline{\mathbb{N}}} \int_{\mathfrak{a}} \int_{\mathbb{K}} F(\bar{n}(\exp H) k) e^{2 \rho(H)} d k d H d \bar{n}
$$

and

$$
\int_{\mathbb{G}} F(g) d g=\int_{\mathbb{K}} \int_{\mathbb{K}} \int_{\mathfrak{a}_{+}} F\left(k_{1}(\exp H) k_{2}\right) \delta(H) d H d k_{1} d k_{2}
$$

for any continuous compactly supported function $F: \mathbb{G} \rightarrow \mathbb{C}$. One has by definition

$$
\delta(H)=C \prod_{\alpha \in \Sigma^{+}}(\sinh \alpha(H))^{m_{\alpha}} .
$$

The Killing form of $\mathfrak{g}$ induces a scalar product $\langle$,$\rangle on \mathfrak{a}$. Denote by $\mathfrak{a}^{*}$ and $\mathfrak{a}_{\mathbb{C}}^{*}$ the real and the complex dual of $\mathfrak{a}$. For any $\lambda \in \mathfrak{a}^{*}$ let $H_{\lambda}$ be the unique element of $\mathfrak{a}$ with the property that $\left\langle H_{\lambda}, H\right\rangle=\lambda(H)$ for any $H \in \mathfrak{a}$. We transfer the scalar product $\langle$,$\rangle to \mathfrak{a}^{*}$ and extend it to a $\mathbb{C}$-bilinear form on $\mathfrak{a}_{\mathbb{C}}^{*}$. The Fourier transform of a smooth compactly supported function $f: \mathbb{X} \rightarrow \mathbb{C}$ is by definition the function $\tilde{f}: \mathfrak{a}_{\mathbb{C}}^{*} \times \mathbb{K} / \mathbb{M} \rightarrow \mathbb{C}$ given by

$$
\widetilde{f}(\lambda, b)=\int_{\mathbb{X}} f(z) e^{(-i \lambda+\rho) A(z, b)} d z
$$

where $A(z, b)$ is an $\mathfrak{a}$-valued analogue of the usual scalar product on Euclidean spaces (see [12, Chapter III]). For any $g \in \mathbb{G}$ and $k \in \mathbb{K}$ one has by definition $A(g \mathbb{K}, k \mathbb{M})=-H\left(g^{-1} k\right)$. If $f$ is $\mathbb{K}$-invariant (i.e., $f(k \cdot z)=f(z)$ for any $k \in \mathbb{K}$ and $z \in \mathbb{X})$, then $\widetilde{f}$ does not depend on $b$. The formula (2.2) becomes

$$
\widetilde{f}(\lambda, b)=\int_{\mathbb{G}} f(g \mathbb{K}) \varphi_{-\lambda}(g) d g
$$

where

$$
\varphi_{\lambda}(g)=\int_{\mathbb{K} / \mathbb{M}} e^{(i \lambda+\rho) A(g \mathbb{K}, b)} d b .
$$

A central result in the theory of convolution operators on semisimple Lie groups is the Kunze-Stein phenomenon, which states that if $p \in[1,2), f \in L^{2}(\mathbb{G})$ and $K \in L^{p}(\mathbb{G})$, then

$$
\|f * K\|_{L^{2}(\mathbb{G})} \leq C_{p}\|f\|_{L^{2}(\mathbb{G})}\|K\|_{L^{p}(\mathbb{G})} .
$$

This inequality was proved by Kunze and Stein [15] in the case when the group $\mathbb{G}$ is $\mathbb{S L}(2, \mathbb{R})$ (and, later on, for other particular groups) and by Cowling [7] in the general case. In [13] Herz noticed that the inequality (2.5) can be sharpened (and its proof greatly simplified) if the kernel $K$ is $\mathbb{K}$-bi-invariant, i.e., $K\left(k_{1} g k_{2}\right)=K(g)$ for any $k_{1}, k_{2} \in \mathbb{K}$ and $g \in \mathbb{G}$. Let ||$* K \|_{L^{p}(\mathbb{G})}$ denote the norm of the convolution operator defined by the kernel $K$ and for any $p \in[1, \infty]$ let $\rho_{p}=|2 / p-1| \rho$. One has the following criterion due to Herz [13]: 
Proposition 2.1. If $1 \leq p \leq \infty$ and $K$ is a $\mathbb{K}$-bi-invariant kernel on $\mathbb{G}$, then

$$
\||* K|\|_{L^{p}(\mathbb{G})} \leq C \int_{\mathbb{G}}|K(g)| \varphi_{-i \rho_{p}}(g) d g .
$$

It is easy to see that if $1 \leq p<2$ and $H \in \overline{\mathfrak{a}^{+}}$(the closure of the cone $\mathfrak{a}^{+}$), then $\varphi_{-i \rho_{p}}(\exp H) \leq C_{p} e^{-2 \rho(H) / p^{\prime}}$, where $p^{\prime}=p /(p-1)$ is the conjugate exponent of $p$. Thus (2.6) becomes

$$
\||| * K||_{L^{p}(\mathbb{G})} \leq C_{p} \int_{\overline{\mathfrak{a}}^{+}}|K(\exp H)| \delta(H) e^{-2 \rho(H) / p^{\prime}} d H .
$$

\section{A Transference Theorem}

Let $\phi: \mathfrak{a} \rightarrow \mathbb{C}$ be a function on $\mathfrak{a}$ supported in $\overline{\mathfrak{a}^{+}}$. Let $\ell=\operatorname{dim} \mathfrak{a}$ denote the rank of the group $\mathbb{G}$. Assume that the function $\phi$ satisfies the following basic assumption: there exist two constants $A$ and $c_{0}>0$ such that

$$
\left|\phi\left(H_{1}\right)-\phi\left(H_{2}\right)\right| \leq A\left(1+\left|H_{1}\right|\right)^{-\left(\ell+c_{0}\right)}
$$

for any $H_{1}, H_{2} \in \overline{\mathfrak{a}^{+}}$with the property that $\left|H_{1}-H_{2}\right| \leq 1$.

Let $p$ be a fixed exponent in the interval $(1,2)$ and let $K_{p, \phi}: \mathbb{G} \rightarrow \mathbb{C}$ be the $\mathbb{K}$-bi-invariant kernel on $\mathbb{G}$ given by

$$
K_{p, \phi}\left(k_{1}(\exp H) k_{2}\right)=e^{-2 \rho(H) / p} \phi(H)
$$

for any $H \in \overline{\mathfrak{a}^{+}}$and $k_{1}, k_{2} \in \mathbb{K}$. As in the previous section, let

$$
\left\|\left|* K_{p, \phi}\right|\right\|_{L^{p}(\mathbb{X})}=\left|\left\|* K_{p, \phi} \mid\right\|_{L^{p}(\mathbb{G})}\right.
$$

denote the norm of the convolution operator with the kernel $K_{p, \phi}$ on $L^{p}(\mathbb{X})$ and $\||| * \phi \mid\|_{L^{p}(\mathfrak{a})}$ the norm of the convolution operator with the kernel $\phi$ on $L^{p}(\mathfrak{a})$. Our first main theorem is the following:

Theorem 3.1. Assume that $\phi$ satisfies (3.1), $p \in(1,2)$, and $K_{p, \phi}$ is defined as in (3.2). Then there exists a constant $C_{p}$ depending only on $p$ and the group $\mathbb{G}$ such that

$$
\||| K_{p, \phi}||_{L^{p}(\mathbb{X})} \leq C_{p}\left(\|\left.|* \phi|\right|_{L^{p}(\mathfrak{a})}+\widetilde{A}\right) .
$$

The constant $\widetilde{A}$ depends only on $\ell$ and the constants $A$ and $c_{0}$ in (3.1).

Remark 1. This theorem is sharper than the classical transference principle of Coifman and Weiss [6, Theorem 8.7] because the factor that makes the transition between the kernels $\phi$ and $K_{p, \phi}$ is $e^{2 \rho(H) / p}$. This transition factor is $\delta(H)$ in [6] Theorem 8.7], which is proportional to $e^{2 \rho(H)}$ if $H$ is not close to the walls of the Weyl chamber $\mathfrak{a}^{+}$. See also [8, Theorem 4.1] and [9] for other transference principles.

As an application, we have a new variant of Herz's majorizing principle. Assume that the function $\phi$ is supported in the cone $\overline{\mathfrak{a}^{+}}$and satisfies the differential inequalities

$$
\left|\partial_{H}^{j} \phi(H)\right| \leq A^{\prime}(1+|H|)^{-\ell-j}
$$

for any $H \in \mathfrak{a}^{+}$and $j=0$ or $j=1$, where $\partial_{H}^{j} \phi(H)$ denotes any partial derivative of $\phi$ of order $j$. Assume also that the function $\phi$ satisfies the cancellation condition

$$
\left|\int_{|H| \leq N} \phi(H) d H\right| \leq A^{\prime}
$$


for any $N>0$. In this case the function $\phi$ is a kernel of the Calderon-Zygmund type on a. Indeed, the conditions (3.3) and (3.4) guarantee the fact that the kernel $\phi$ defines a bounded operator on $L^{q}(\mathfrak{a})$ for any $q \in(1, \infty)$ (see, for example, [20. Chapters I and VI]). One has the following consequence of Theorem 3.1

Corollary 3.2. If $p \in(1,2)$ and $\phi$ satisfies (3.3) and (3.4), then

$$
\left\|* K_{p, \phi}\right\|_{L^{p}(\mathbb{G})} \leq C_{p}
$$

The constant $C_{p}$ depends only on $p$ and $A^{\prime}$.

Notice that, in general, the function $\phi$ in Corollary 3.2 is not absolutely integrable at $\infty$; therefore, this corollary cannot be obtained as a consequence of the KunzeStein phenomenon or Herz majorizing principle. Easy examples, similar to the ones in [14, show that the large scale cancellation condition (3.4) is crucial. We also remark that both Theorem 3.1 and Corollary 3.2 are false if $p=2$.

The rest of this section is devoted to proving Theorem 3.1 We will follow closely the line of the proof of Theorem 1 in [14]. Throughout the proof of Theorem 3.1 the letter $C$ will denote universal constants depending only on the group $\mathbb{G}, C_{p}$ will denote constants depending on $p$ and the group $\mathbb{G}$, and $\widetilde{A}$ will denote constants depending only on $\ell$ and the values of $c_{0}$ and $A$ in (3.1). Elements of $\mathfrak{a}$ will be denoted by $H, H_{1}$, and $H_{2}$, and elements of $\overline{\mathbb{N}}$ will be denoted by $\bar{n}, \bar{m}$, and $\bar{v}$.

Recall that for any locally integrable $\mathbb{K}$-bi-invariant function $K$ and any smooth compactly supported function $f: \mathbb{X} \rightarrow \mathbb{C}$ the convolution $f * K$ is defined by the formula

$$
f * K(z)=\int_{\mathbb{G}} f(h \cdot \mathbf{0}) K\left(h^{-1} \cdot z\right) d h
$$

where $\mathbf{0}=\mathbb{G} / \mathbb{K}$ is the origin of the symmetric space $\mathbb{X}$. Notice that

$$
\left\|\left|* K_{p, \phi}\|\|_{L^{p}(\mathbb{X})}=\sup _{\|f\|_{p}=\|g\|_{p^{\prime}}=1}\right| \int_{\mathbb{G}} \int_{\mathbb{G}} f(h \cdot \mathbf{0}) K_{p, \phi}\left(h^{-1} h^{\prime}\right) g\left(h^{\prime} \cdot \mathbf{0}\right) d h d h^{\prime} \mid,\right.
$$

where the supremum is taken over smooth compactly supported functions $f, g$ : $\mathbb{X} \rightarrow \mathbb{C}$, and $p^{\prime}=p /(p-1)$ is the conjugate exponent of $p$.

As in the proof of Theorem 1 in [14, the main idea is to estimate integrals in Iwasawa coordinates. We identify the group $\mathbb{G}$ with $\overline{\mathbb{N}} \times \mathfrak{a} \times \mathbb{K}$ using the Iwasawa decomposition $\mathbb{G}=\overline{\mathbb{N}}(\exp \mathfrak{a}) \mathbb{K}$. Let $d \mu$ be the measure on $\overline{\mathbb{N}} \times \mathfrak{a}$ corresponding to this decomposition, i.e., $d \mu=e^{2 \rho(H)} d \bar{n} d H$. Thus it suffices to prove that for any smooth compactly supported functions $f, g: \overline{\mathbb{N}} \times \mathfrak{a} \rightarrow \mathbb{C}$ one has

$$
\left|I_{p, \phi}(f, g)\right| \leq C_{p}\left(\left|\|* \phi \mid\|_{L^{p}(\mathfrak{a})}+\widetilde{A}\right)\|f\|_{L^{p}(\overline{\mathbb{N}} \times \mathfrak{a}, d \mu)}\|g\|_{L^{p^{\prime}}(\overline{\mathbb{N}} \times \mathfrak{a}, d \mu)}\right.
$$

where

$$
\begin{aligned}
I_{p, \phi}(f, g)=\int_{\overline{\mathbb{N}}} \int_{\overline{\mathbb{N}}} \int_{\mathfrak{a}} \int_{\mathfrak{a}} f\left(\bar{m}, H_{1}\right) K_{p, \phi} & \left(\delta_{-H_{1}}\left(\bar{m}^{-1} \bar{n}\right)\left(\exp \left(H_{2}-H_{1}\right)\right)\right) \\
& \times g\left(\bar{n}, H_{2}\right) e^{2 \rho\left(H_{1}+H_{2}\right)} d H_{1} d H_{2} d \bar{m} d \bar{n} .
\end{aligned}
$$

By definition $\delta_{H}(\bar{v})=(\exp H) \bar{v}(\exp -H)$ for any $H \in \mathfrak{a}$ and $\bar{v} \in \overline{\mathbb{N}}$. It is clear that $\delta_{H}$ is a dilation of the group $\overline{\mathbb{N}}$. In order to estimate $\left|I_{p, \phi}(f, g)\right|$ we need to understand the connection between the Iwasawa decomposition and the Cartan decomposition of the group $\mathbb{G}$. Let ${ }^{+} \mathfrak{a}$ denote the interior of the cone $\{H \in \mathfrak{a}$ : 
$\left\langle H, H^{\prime}\right\rangle>0$ for any $\left.H^{\prime} \in \mathfrak{a}^{+}\right\}$. It is well known that $\mathfrak{a}^{+} \subset{ }^{+} \mathfrak{a}$ (see 10, Lemma 35]). For any $H \in \mathfrak{a}^{+}$let

$$
\omega(H)=\min _{\alpha \in \Sigma^{+}} \alpha(H) .
$$

Lemma 3.3. If $\bar{v} \in \overline{\mathbb{N}}$ and $H \in \mathfrak{a}^{+}$, then

$$
[\bar{v}(\exp H)]^{+}=H+H(\bar{v})+H^{\prime}(\bar{v}, H)
$$

where $H^{\prime}(\bar{v}, H) \in \overline{{ }^{+} \mathfrak{a}}$ and there exists a constant $C_{0}$ such that

$$
e^{2 \rho\left(H^{\prime}(\bar{v}, H)\right)}-1 \leq C e^{-2 \omega(H)} e^{C_{0} \rho(H(\bar{v}))} .
$$

Proof of Lemma 3.3 . This lemma is a quantitative version of Proposition 4.24 in 12, Chapter II]. Recall that $g^{+}$and $H(g)$ denote the a-components of the element $g \in \mathbb{G}$ in the Cartan decomposition and the Iwasawa decomposition of the group $\mathbb{G}$. It is well known that $g^{+}-H(g) \in \overline{{ }^{+} \mathfrak{a}}$ for any $g \in \mathbb{G}([10$, Lemma 35$])$; therefore $H^{\prime}(\bar{v}, H) \in \overline{{ }^{\prime}}$. The estimate (3.8) is a consequence of the proof of Proposition 4.24 in [12. Chapter II]. With the notation in this proposition, let $\pi$ be an irreducible representation of $\mathbb{G}$ on a finite-dimensional vector space $V, \Lambda$ the highest weight of $\pi$, and $\nu$ the restriction of $\Lambda$ to $\mathfrak{a}$. Let

$$
V=V_{\nu}+\sum_{\mu<\nu} V_{\mu}=V_{\nu}+W
$$

be the decomposition of $V$ into weight subspaces. Fix $v_{1}, \ldots, v_{r}$ and $w_{1}, \ldots, w_{p}$, the corresponding orthogonal basis consisting of restricted weight vectors. It follows immediately from one of the equations in Proposition 4.24 in [12, Chapter II] that

$$
r\left(e^{2 \nu\left(H^{\prime}(\bar{v}, H)\right)}-1\right) \leq \sum_{j=1}^{p}\left\|\pi(\bar{v}) w_{j}\right\|^{2} e^{\left(2 \mu_{j}-2 \nu\right)(H)-2 \nu(H(\bar{v}))} .
$$

One clearly has

$$
\left\|\pi(\bar{v}) w_{j}\right\| \leq e^{\nu\left(\bar{v}^{+}\right)} .
$$

Therefore, it suffices to prove that for any $\bar{v} \in \overline{\mathbb{N}}$,

$$
\rho\left(\bar{v}^{+}\right) \leq C+C_{0} \rho(H(\bar{v})) .
$$

Notice first that this inequality follows from the explicit formulae in 12, Chapter II, Theorem 6.1] if the group $\mathbb{G}$ has real rank one. One can also take $C_{0}=1$ in this case. In the general case, let $X_{1}, X_{2}, \ldots, X_{r}$ be a basis of the Lie algebra $\overline{\mathfrak{n}}$ with the following properties:

(i) for any $1 \leq j \leq r$, the vector $X_{j}$ belongs to a root space $\mathfrak{g}_{-\alpha}$ for some root $\alpha \in \Sigma^{+}$

(ii) if $\overline{\mathfrak{n}}_{j}$ denotes the linear span of the vectors $X_{1}, \ldots, X_{j}$, then $\overline{\mathfrak{n}}_{j}$ is an ideal of $\overline{\mathfrak{n}}$ for any $1 \leq j \leq r$.

The map $\bar{v}: \mathbb{R}^{r} \rightarrow \overline{\mathbb{N}}$,

$$
\bar{v}\left(x_{1}, \ldots, x_{r}\right)=\left(\exp x_{r} X_{r}\right) \cdots\left(\exp x_{1} X_{1}\right)
$$

gives a parametrization of the group $\overline{\mathbb{N}}$. For any $\bar{v}=\bar{v}\left(x_{1}, \ldots, x_{r}\right) \in \overline{\mathbb{N}}$ let

$$
\|\bar{v}\|=\max _{j}\left|x_{j}\right|
$$


A similar "norm" function on $\overline{\mathbb{N}}$ was used in [16]. It follows from [16, Lemma 5.3] that there exists a constant $c_{1}>0$ such that

$$
e^{-2 \rho(H(\bar{v}))} \leq C(2+\|\bar{v}\|)^{-c_{1}} .
$$

Thus

$$
\log (2+\|\bar{v}\|) \leq C+C_{1} \rho(H(\bar{v})) .
$$

We will now show that

$$
\left|\bar{v}^{+}\right| \leq C \log (2+\|\bar{v}\|)
$$

Let $d($,$) denote the distance function on the symmetric space \mathbb{X}$ induced by the Killing form on $\mathfrak{g}$. One has $\left|\bar{v}^{+}\right|=d(\bar{v} \mathbb{K}, e \mathbb{K})$. Therefore, if $\bar{v}=\bar{v}\left(x_{1}, \ldots, x_{r}\right)$, then

$$
\left|\bar{v}^{+}\right| \leq \sum_{j=1}^{r} d\left(\left(\exp x_{j} X_{j}\right) \mathbb{K}, e \mathbb{K}\right) .
$$

It follows from the rank-one reduction method and the explicit rank-one formulae in [12, Chapter II, Theorem 6.1] that

$$
d\left(\left(\exp x_{j} X_{j}\right) \mathbb{K}, e \mathbb{K}\right) \leq C \log \left(2+\left|x_{j}\right|\right) .
$$

Thus (3.11) follows from these last two estimates and (3.9) follows from (3.10), (3.11), and the observation that $\rho\left(\bar{v}^{+}\right) \leq|\rho|\left|\bar{v}^{+}\right|$.

We will use this lemma to estimate the function $(\bar{v}, H) \rightarrow K_{p, \phi}(\bar{v}(\exp H))$ for any $\bar{v} \in \overline{\mathbb{N}}$ and $H \in \overline{\mathfrak{a}^{+}}$. Let $P(\bar{v})=e^{-\rho(H(\bar{v}))}$; it is well known (see [10, Lemma 45]) that for any $\varepsilon>0$,

$$
\int_{\overline{\mathbb{N}}} P(\bar{v})^{(1+\varepsilon)} d \bar{v}=C_{\varepsilon}<\infty .
$$

Lemma 3.4. (i) If $H \in \overline{\mathfrak{a}^{+}}$and $\bar{v} \in \overline{\mathbb{N}}$, then

$$
\left|K_{p, \phi}(\bar{v}(\exp H))\right| \leq \widetilde{A} e^{-2 \rho(H) / p}(1+|H|)^{-\left(\ell-1+c_{0}\right)} P(\bar{v})^{2 / p} .
$$

(ii) If $H \in \overline{\mathfrak{a}^{+}}$and $\bar{v} \in \overline{\mathbb{N}}$, then

$$
K_{p, \phi}(\bar{v}(\exp H))=e^{-2 \rho(H) / p} \phi(H) P(\bar{v})^{2 / p}+E_{p, \phi}(\bar{v}, H)
$$

where

$$
\begin{aligned}
\left|E_{p, \phi}(\bar{v}, H)\right| \leq C \cdot \widetilde{A} e^{-2 \rho(H) / p} & (1+|H|)^{-\left(\ell-1+c_{0}\right)} P(\bar{v})^{2 / p} \\
& \times\left(\frac{1+\rho(H(\bar{v}))}{1+|H|}+\min \left(1, e^{-2 \omega(H)} e^{C_{0} \rho(H(\bar{v}))}\right)\right) .
\end{aligned}
$$

The constant $C_{0}$ is the same as in Lemma 3.3 .

Proof of Lemma[3.4. Notice first that

$$
\lim _{|H| \rightarrow \infty} \phi(H)=0 .
$$

This follows immediately from (3.1) and the fact that $\left|\|* \phi \mid\|_{L^{p}(\mathfrak{a})}\right.$ is finite. Thus it follows from (3.1) that

$$
|\phi(H)| \leq \widetilde{A}(1+|H|)^{-\left(\ell-1+c_{0}\right)}
$$


for any $H \in \overline{\mathfrak{a}^{+}}$. To prove part (i) of the proposition notice that

$$
K_{p, \phi}(\bar{v}(\exp H))=e^{-2 \rho\left(H+H(\bar{v})+H^{\prime}(\bar{v}, H)\right) / p} \phi\left(H+H(\bar{v})+H^{\prime}(\bar{v}, H)\right) .
$$

Recall that $H(\bar{v})$ and $H^{\prime}(\bar{v}, H)$ belong to $\overline{{ }^{\mathfrak{a}}}([10$, Lemma 43]). Also, it is well known that $\rho(\overline{+} \mathfrak{a})>0$, i.e., $H_{\rho} \in \mathfrak{a}^{+}$. Thus (3.13) follows easily from (3.16).

To prove part (ii) notice that it follows from Lemma 3.3 that the difference between $K_{p, \phi}(\bar{v}(\exp H))$ and $e^{-2 \rho(H) / p} \phi(H) P(\bar{v})^{2 / p}$ is equal to

$$
e^{-2 \rho(H) / p} P(\bar{v})^{2 / p}\left[e^{-2 \rho\left(H^{\prime}(\bar{v}, H)\right) / p} \phi\left(H+H(\bar{v})+H^{\prime}(\bar{v}, H)\right)-\phi(H)\right] .
$$

Since $H(\bar{v})$ and $H^{\prime}(\bar{v}, H)$ belong to $\overline{{ }^{+} \mathfrak{a}}$, one has

$$
\left|H(\bar{v})+H^{\prime}(\bar{v}, H)\right| \leq C \rho(H(\bar{v}))+\rho\left(H^{\prime}(\bar{v}, H)\right) .
$$

One can now combine the estimates (3.8) and (3.16) and the basic inequality (3.1) to show that

$$
\begin{aligned}
\left|E_{p, \phi}(\bar{v}, H)\right| \leq C \cdot \widetilde{A} e^{-2 \rho(H) / p}(1+|H|)^{-\left(\ell+c_{0}\right)} P(\bar{v})^{2 / p} & \\
& \times\left[1+\rho(H(\bar{v}))+e^{-2 \omega(H)}(1+|H|) e^{C_{0} \rho(H(\bar{v}))}\right] .
\end{aligned}
$$

In addition, it follows from (3.13) and (3.16) that

$$
\left|E_{p, \phi}(\bar{v}, H)\right| \leq \widetilde{A} e^{-2 \rho(H) / p}(1+|H|)^{-\left(\ell-1+c_{0}\right)} P(\bar{v})^{2 / p}
$$

and the estimate (3.15) follows from the last two inequalities.

We return to the proof of Theorem 3.1. Let $\chi$ be the characteristic function of the set $\overline{\mathfrak{a}^{+}}$; we decompose the integral $I_{p, \phi}(f, g)$ in (3.6) as $I_{p, \phi}(f, g)=I_{p, \phi}^{1}(f, g)+$ $I_{p, \phi}^{2}(f, g)+I_{p, \phi}^{3}(f, g)$, where

$$
\begin{gathered}
I_{p, \phi}^{1}(f, g)=\int_{\overline{\mathbb{N}}} \int_{\overline{\mathbb{N}}} \int_{\mathfrak{a}} \int_{\mathfrak{a}} f\left(\bar{m}, H_{1}\right) K_{p, \phi}\left(\delta_{-H_{1}}\left(\bar{m}^{-1} \bar{n}\right)\left(\exp \left(H_{2}-H_{1}\right)\right)\right) \\
\times g\left(\bar{n}, H_{2}\right) e^{2 \rho\left(H_{1}+H_{2}\right)}\left(1-\chi\left(H_{2}-H_{1}\right)\right) d H_{1} d H_{2} d \bar{m} d \bar{n}, \\
I_{p, \phi}^{2}(f, g)=\int_{\overline{\mathbb{N}}} \int_{\overline{\mathbb{N}}} \int_{\mathfrak{a}} \int_{\mathfrak{a}} f\left(\bar{m}, H_{1}\right) E_{p, \phi}\left(\delta_{-H_{1}}\left(\bar{m}^{-1} \bar{n}\right),\left(H_{2}-H_{1}\right)\right) \\
\times g\left(\bar{n}, H_{2}\right) e^{2 \rho\left(H_{1}+H_{2}\right)} \chi\left(H_{2}-H_{1}\right) d H_{1} d H_{2} d \bar{m} d \bar{n},
\end{gathered}
$$

and

$$
\begin{aligned}
I_{p, \phi}^{3}(f, g)=\int_{\overline{\mathbb{N}}} \int_{\overline{\mathbb{N}}} \int_{\mathfrak{a}} \int_{\mathfrak{a}} f\left(\bar{m}, H_{1}\right) & P\left(\delta_{-H_{1}}\left(\bar{m}^{-1} \bar{n}\right)\right) e^{-2 \rho\left(H_{2}-H_{1}\right) / p} \phi\left(H_{2}-H_{1}\right) \\
& \times g\left(\bar{n}, H_{2}\right) e^{2 \rho\left(H_{1}+H_{2}\right)} \chi\left(H_{2}-H_{1}\right) d H_{1} d H_{2} d \bar{m} d \bar{n} .
\end{aligned}
$$

We record an elementary fact that will be used several times in the rest of the paper.

Lemma 3.5. One has

$$
\int_{\overline{\mathfrak{a}^{+}}}(1+|H|)^{-\left(\ell-1+\varepsilon_{0}\right)} e^{-\varepsilon_{1} \omega(H)} d H \leq C_{\varepsilon_{0}, \varepsilon_{1}}
$$

for any $\varepsilon_{0}, \varepsilon_{1}>0$.

Lemma 3.6. One has

$$
\left|I_{p, \phi}^{1}(f, g)\right| \leq C_{p} \cdot \widetilde{A}|| f\left\|_{L^{p}(\overline{\mathbb{N}} \times \mathfrak{a}, d \mu)}\right\| g \|_{L^{p^{\prime}(\bar{N} \times \mathfrak{a}, d \mu)}} .
$$


Proof of Lemma 3.6 . Let

$$
\left\{\begin{array}{l}
F_{1}\left(H_{1}\right)=\left[\int_{\overline{\mathbb{N}}}\left|f\left(\bar{m}, H_{1}\right)\right|^{p} d \bar{m}\right]^{1 / p} \\
G_{1}\left(H_{2}\right)=\left[\int_{\overline{\mathbb{N}}}\left|g\left(\bar{n}, H_{2}\right)\right|^{p^{\prime}} d \bar{n}\right]^{1 / p^{\prime}} .
\end{array}\right.
$$

By Hölder's inequality, the absolute value of $I_{p, \phi}^{1}(f, g)$ is dominated by

$$
\begin{aligned}
\int_{\mathfrak{a}} \int_{\mathfrak{a}} F_{1}\left(H_{1}\right) G_{1}\left(H_{2}\right) e^{2 \rho\left(H_{1}+H_{2}\right)}\left(1-\chi\left(H_{2}-H_{1}\right)\right) \\
\times\left(\int_{\overline{\mathbb{N}}}\left|K_{p, \phi}\left(\delta_{-H_{1}}(\bar{v})\left(\exp \left(H_{2}-H_{1}\right)\right)\right)\right| d \bar{v}\right) d H_{1} d H_{2} .
\end{aligned}
$$

Recall that the map $\bar{n}_{1} \rightarrow \bar{n}_{2}=\delta_{H}\left(\bar{n}_{1}\right)$ is a dilation of $\overline{\mathbb{N}}$ with $d \bar{n}_{2}=e^{-2 \rho(H)} d \bar{n}_{1}$. In addition, it is well known that the Abel transform

$$
\mathcal{A}_{2} k(H)=e^{\rho(H)} \int_{\overline{\mathbb{N}}} k(\bar{v}(\exp H)) d \bar{v}
$$

takes suitable $\mathbb{K}$-bi-invariant functions $k$ to $W$-invariant functions on $\mathfrak{a}$. For any regular element $H \in \mathfrak{a}$ let $H^{+} \in \mathfrak{a}^{+}$be its representative in the positive Weyl chamber, i.e., $H^{+} \in(W \cdot H) \cap \mathfrak{a}^{+}$. Thus, if $H \in \mathfrak{a}$ is regular, one has

$$
\begin{aligned}
\int_{\overline{\mathbb{N}}}\left|K_{p, \phi}(\bar{v}(\exp H))\right| d \bar{v} & =e^{\rho\left(H^{+}-H\right)} \int_{\overline{\mathbb{N}}}\left|K_{p, \phi}\left(\bar{v}\left(\exp H^{+}\right)\right)\right| d \bar{v} \\
& \leq C_{p} \cdot \widetilde{A} e^{\rho\left(H^{+}\right)} e^{-\rho(H)} e^{-2 \rho\left(H^{+}\right) / p}(1+|H|)^{-\left(\ell-1+c_{0}\right)} .
\end{aligned}
$$

The last estimate is a consequence of (3.12) and (3.13). It follows after several simplifications that the absolute value of the integral in (3.20) is dominated by

$$
\begin{aligned}
C_{p} \cdot \widetilde{A} \int_{\mathfrak{a}} \int_{\mathfrak{a}} F_{1}\left(H_{1}\right) G_{1}\left(H_{2}\right) & \left(1-\chi\left(H_{2}-H_{1}\right)\right)\left(1+\left|H_{2}-H_{1}\right|\right)^{-\left(\ell-1+c_{0}\right)} \\
& \times e^{-\rho\left(\left(H_{2}-H_{1}\right)^{+}\right)(2 / p-1)} e^{\rho\left(H_{1}+H_{2}\right)} d H_{1} d H_{2} .
\end{aligned}
$$

The change of variable $H_{2}=H_{1}+H$ shows that this integral is equal to

$$
\begin{aligned}
C_{p} \cdot \widetilde{A} \int_{\mathfrak{a}}\left(\int_{\mathfrak{a}}(\right. & \left.\left.F_{1}\left(H_{1}\right) e^{2 \rho\left(H_{1}\right) / p}\right)\left(G_{1}\left(H_{1}+H\right) e^{2 \rho\left(H_{1}+H\right) / p^{\prime}}\right) d H_{1}\right) \\
& \times(1-\chi(H))(1+|H|)^{-\left(\ell-1+c_{0}\right)} e^{-\rho\left(H^{+}-H\right)(2 / p-1)} d H .
\end{aligned}
$$

By Hölder's inequality, the inner integral is dominated by

$$
\left(\int_{\mathfrak{a}}\left|F_{1}\left(H_{1}\right)\right|^{p} e^{2 \rho\left(H_{1}\right)} d H_{1}\right)^{1 / p}\left(\int_{\mathfrak{a}}\left|G_{1}\left(H_{2}\right)\right|^{p^{\prime}} e^{2 \rho\left(H_{2}\right)} d H_{2}\right)^{1 / p^{\prime}}
$$

which is equal to

$$
\left.\|f\|_{L^{p}(\overline{\mathbb{N}} \times \mathfrak{a}, d \mu)}|| g\right|_{L^{p^{\prime}(\overline{\mathbb{N}} \times \mathfrak{a}, d \mu)}} .
$$

Thus one only needs to estimate

$$
\int_{\mathfrak{a}}(1-\chi(H))(1+|H|)^{-\left(\ell-1+c_{0}\right)} e^{-\rho\left(H^{+}-H\right)(2 / p-1)} d H .
$$

Notice that if $H \notin \overline{\mathfrak{a}^{+}}$, then $\rho\left(H^{+}-H\right) \geq \omega(H)$, where $\omega(H)$ has the same meaning as in Lemma 3.3 Since $p<2$, it follows from Lemma 3.5 that the integral in (3.21) is dominated by $C_{p} \cdot \widetilde{A}$ and the lemma follows. 
Lemma 3.7. One has

$$
\left|I_{p, \phi}^{2}(f, g)\right| \leq C_{p} \cdot \widetilde{A}|| f\left\|_{L^{p}(\overline{\mathbb{N}} \times \mathfrak{a}, d \mu)}\right\| g \|_{L^{p^{\prime}(\bar{N} \times \mathfrak{a}, d \mu)}} .
$$

Proof of Lemma[3.7. By Hölder's inequality, the absolute value of the integral $I_{p, \phi}^{2}(f, g)$ is dominated by

$$
\begin{aligned}
\int_{\mathfrak{a}} \int_{\mathfrak{a}} F_{1}\left(H_{1}\right) G_{1}\left(H_{2}\right) e^{2 \rho\left(H_{1}+H_{2}\right)} \chi\left(H_{2}-H_{1}\right) \\
\times\left(\int_{\overline{\mathbb{N}}}\left|E_{p, \phi}\left(\delta_{-H_{1}}(\bar{v}),\left(H_{2}-H_{1}\right)\right)\right| d \bar{v}\right) d H_{1} d H_{2} .
\end{aligned}
$$

Notice first that

$$
\int_{\overline{\mathbb{N}}}\left|E_{p, \phi}\left(\delta_{-H_{1}}(\bar{v}),\left(H_{2}-H_{1}\right)\right)\right| d \bar{v}=e^{-2 \rho\left(H_{1}\right)} \int_{\overline{\mathbb{N}}}\left|E_{p, \phi}\left(\bar{v},\left(H_{2}-H_{1}\right)\right)\right| d \bar{v} .
$$

One can use (3.15) to estimate the integral of $E_{p, \phi}$. It follows from (3.12) that

$$
\int_{\overline{\mathbb{N}}} P(\bar{v})^{2 / p}\left(1+\rho(H(\bar{v})) d \bar{v} \leq C_{p} .\right.
$$

Also

$$
\begin{aligned}
\int_{\overline{\mathbb{N}}} P(\bar{v})^{2 / p} & \min \left(1, e^{-2 \omega(H)} e^{C_{0} \rho(H(\bar{v}))}\right) d \bar{v} \\
& \leq e^{-\omega(H)} \int_{\rho(H(\bar{v})) \leq \omega(H) / C_{0}} P(\bar{v})^{2 / p} d \bar{v}+\int_{\rho(H(\bar{v})) \geq \omega(H) / C_{0}} P(\bar{v})^{2 / p} d \bar{v} \\
& \leq C_{p} e^{-\omega(H)}+\int_{\overline{\mathbb{N}}} e^{-(1 / 2+1 / p) \rho(H(\bar{v}))} e^{-(1 / p-1 / 2) \omega(H) / C_{0}} d \bar{v} \\
& \leq C_{p} e^{-c_{p} \omega(H)}
\end{aligned}
$$

where $c_{p}=(1 / p-1 / 2) / C_{0}>0$. It follows from the last two estimates and (3.15) that if $H \in \overline{\mathfrak{a}^{+}}$, then

$$
\int_{\overline{\mathbb{N}}}\left|E_{p, \phi}(\bar{v}, H)\right| d \bar{v} \leq C_{p} \cdot \widetilde{A} e^{-2 \rho(H) / p} \Psi(H)
$$

where

$$
\Psi(H)=(1+|H|)^{-\left(\ell+c_{0}\right)}+(1+|H|)^{-\left(\ell-1+c_{0}\right)} e^{-c_{p} \omega(H)} .
$$

Let $\Psi(H)=0$ if $H \notin \overline{\mathfrak{a}^{+}}$. It follows from (3.18) that $\Psi \in L^{1}(\mathfrak{a})$, i.e.,

$$
\|\Psi\|_{L^{1}(\mathfrak{a})} \leq C_{p} \cdot \widetilde{A}
$$

One substitutes the estimate (3.24) in (3.23). The change of variable $H_{2}=H_{1}+H$ shows that the absolute value of $I_{p, \phi}^{2}(f, g)$ is dominated by

$$
C_{p} \cdot \widetilde{A} \int_{\mathfrak{a}}\left(\int_{\mathfrak{a}}\left(F_{1}\left(H_{1}\right) e^{2 \rho\left(H_{1}\right) / p}\right)\left(G_{1}\left(H_{1}+H\right) e^{2 \rho\left(H_{1}+H\right) / p^{\prime}}\right) d H_{1}\right) \chi(H) \Psi(H) d H .
$$

The lemma now follows from this last estimate, Hölder's inequality and (3.25).

Lemma 3.8. One has

$$
\left|I_{p, \phi}^{3}(f, g)\right| \leq C_{p}|||* \phi|\left\|_{L^{p}(\mathfrak{a})}\right\| f\left\|_{L^{p}(\overline{\mathbb{N}} \times \mathfrak{a}, d \mu)}\right\| g \|_{L^{p^{\prime}(\overline{\mathbb{N}} \times \mathfrak{a}, d \mu)}} .
$$


Proof of Lemma[3.8. The change of variable $H_{2}=H_{1}+H$ shows that $I_{p, \phi}^{3}(f, g)$ is equal to

$$
\int_{\overline{\mathbb{N}}} \int_{\overline{\mathbb{N}}} \int_{\mathfrak{a}} f\left(\bar{m}, H_{1}\right) G_{2}\left(\bar{n}, H_{1}\right) P\left(\delta_{-H_{1}}\left(\bar{m}^{-1} \bar{n}\right)\right)^{2 / p} e^{2 \rho\left(H_{1}\right)} e^{2 \rho\left(H_{1}\right) / p} d H_{1} d \bar{m} d \bar{n}
$$

where

One has

$$
G_{2}\left(\bar{n}, H_{1}\right)=\int_{\mathfrak{a}} g\left(\bar{n}, H_{1}+H\right) e^{2 \rho\left(H_{1}+H\right) / p^{\prime}} \phi(H) d H
$$

$$
\left(\int_{\mathfrak{a}}\left|G_{2}\left(\bar{n}, H_{1}\right)\right|^{p^{\prime}} d H_{1}\right)^{1 / p^{\prime}} \leq\left|\|* \phi \mid\|_{L^{p}(\mathfrak{a})}\left(\int_{\mathfrak{a}}\left|g\left(\bar{n}, H_{2}\right)\right|^{p^{\prime}} e^{2 \rho\left(H_{2}\right)} d H_{2}\right)^{1 / p^{\prime}} .\right.
$$

Let

$$
G_{3}\left(H_{1}\right)=\left(\int_{\bar{N}}\left|G_{2}\left(\bar{n}, H_{1}\right)\right|^{p^{\prime}} d \bar{n}\right)^{1 / p^{\prime}} .
$$

It follows by Hölder's inequality and (3.12) that the absolute value of the integral (3.27) is dominated by

$$
\int_{\mathfrak{a}} F_{1}\left(H_{1}\right) G_{3}\left(H_{1}\right) e^{2 \rho\left(H_{1}\right) / p} d H_{1},
$$

which, again by Hölder's inequality, is dominated by

$$
\left(\int_{\mathfrak{a}} G_{3}\left(H_{1}\right)^{p^{\prime}} d H_{1}\right)^{1 / p^{\prime}}\left(\int_{\mathfrak{a}} F_{1}\left(H_{1}\right)^{p} e^{2 \rho\left(H_{1}\right)} d H_{1}\right)^{1 / p} .
$$

The estimate (3.26) follows.

The main estimate (3.5) is a consequence of (3.19), (3.22) and (3.26).

\section{4. $L^{p}$ Fourier Multipliers}

Recall that the Fourier transform on the symmetric space $\mathbb{X}$ associates to any smooth compactly supported function $f$ on $\mathbb{X}$ a function $\tilde{f}: \mathfrak{a}_{\mathbb{C}}^{*} \times \mathbb{K} / \mathbb{M} \rightarrow \mathbb{C}$. By the Plancherel theorem, any bounded $W$-invariant multiplier $m: \mathfrak{a}^{*} \rightarrow \mathbb{C}$ defines a bounded operator $T_{m}$ on $L^{2}(\mathbb{X})$ given by $\widetilde{T_{m} f}(\lambda, b)=m(\lambda) \tilde{f}(\lambda, b)$. Assume that $p \in(1,2) \cup(2, \infty)$ and let $\rho_{p}=|2 / p-1| \rho$. Let

$$
\mathcal{T}_{p}=\mathfrak{a}^{*}+i \operatorname{co}\left(W \cdot \rho_{p}\right)
$$

where $\operatorname{co}\left(W \cdot \rho_{p}\right)$ denotes the interior of the convex hull of the set of points $\left\{w \cdot \rho_{p}\right.$ : $w \in W\}$.

Assume that one has a multiplier $m: \mathfrak{a}^{*} \rightarrow \mathbb{C}$ that extends to a bounded $W$ invariant holomorphic function in the interior of the tube $\mathcal{T}_{p}$. Assume, in addition, that $m$ satisfies the differential inequalities

$$
\left|\partial_{\lambda}^{j} m(\lambda)\right| \leq A_{j} d(\lambda)^{-j}
$$

for any $j=0,1, \ldots$ and $\lambda \in \mathcal{T}_{p}$. Here $d(\lambda)$ denotes the distance between the point $\lambda \in \mathcal{T}_{p}$ and the set $i\left(\mathfrak{a}^{*} \backslash \operatorname{co}\left(W \cdot \rho_{p}\right)\right)$. More precisely, if $\lambda=\eta+i \xi$, then $d(\lambda)=\left(|\eta|^{2}+d\left(\xi, \mathfrak{a}^{*} \backslash \operatorname{co}\left(W \cdot \rho_{p}\right)\right)^{2}\right)^{1 / 2}$. As before, $\partial_{\lambda}^{j} m(\lambda)$ denotes any partial derivative of $m$ of order $j$. 
Theorem 4.1. If $p \in(1,2) \cup(2, \infty)$ and $m$ satisfies the differential inequalities (4.1), then the operator $T_{m}$ extends to a bounded operator on $L^{p}(\mathbb{X})$.

An operator $T_{m}$ defined by a multiplier $m$ that satisfies (4.1) can be thought of as a singular integral operator on the symmetric space $\mathbb{X}$. The rest of this section will be devoted to proving Theorem 4.1. The letter $C$ will denote constants that may depend on the group $\mathbb{G}$ and finitely many of the constants $A_{j}$ in (4.1), and $C_{p}$ will denote constants that may also depend on $p$.

One can assume that $p \in(1,2)$. In order to insure the convergence of the integrals throughout this section we will also assume that the multiplier $m(\lambda)$ is premultiplied with a factor of the form $e^{-\delta\langle\lambda, \lambda\rangle}$, where $0<\delta \leq 1$. Our estimates will be uniform in $\delta$; once one proves suitable uniform estimates, standard limiting arguments allow one to pass to the general theorem. The multiplier $m$ is holomorphic in the interior of the tube $\mathcal{T}_{p}$; therefore we can assume that for any $\xi \in \operatorname{co}\left(W \cdot \rho_{p}\right)$ the function $\eta \rightarrow m(\eta+i \xi)$ is a Schwartz function on $\mathfrak{a}^{*}$.

Let $K$ be the $\mathbb{K}$-bi-invariant kernel of the operator $T_{m}$. By the inversion formula one has

$$
K(\exp H)=C \int_{\mathfrak{a}^{*}} m(\lambda) \varphi_{\lambda}(\exp H)|\mathbf{c}(\lambda)|^{-2} d \lambda
$$

for any $H \in \overline{\mathfrak{a}^{+}}$. The spherical functions $\varphi_{\lambda}$ are defined in (2.4) and $\mathbf{c}$ is the Harish-Chandra function. The idea of the proof is the following. Using smooth cutoff functions we will break up this kernel into three parts. The first part is supported near the origin of the group $\mathbb{G}$ (i.e., in the set $\{\mathbb{K}(\exp H) \mathbb{K}:|H| \leq 2\}$ ). The analysis of this local part of the kernel is contained in [1, Section 4]. The second part of the kernel is supported along the walls of the positive Weyl chamber, i.e., in the set $\left\{\mathbb{K}(\exp H) \mathbb{K}: H \in \overline{\mathfrak{a}^{+}}\right.$and $\left.\omega(H) \leq 2\right\}$. One can use the Herz majorizing principle (see Proposition 2.1 and (2.7)) together with the main idea in 1, Section 2] to deal with this part of the kernel. Finally, the main part of the kernel is supported away from the walls of the positive Weyl chamber, i.e., in the set $\{\mathbb{K}(\exp H) \mathbb{K}:|H| \geq 1$ and $\omega(H) \geq 1\}$. The Herz majorizing principle fails to prove boundedness on $L^{p}(\mathbb{X})$ of the operator defined by this part of the kernel if the multiplier $m$ has a singularity at the point $i \rho_{p}$. We use Theorem 3.1 and the Harish-Chandra expansion of the spherical functions $\varphi_{\lambda}$ to control the norm of the operator defined by this part of the kernel.

Let $\psi_{1}: \overline{\mathfrak{a}^{+}} \rightarrow[0,1]$ be a smooth cutoff function such that $\psi_{1}(H)=0$ if $|H| \leq 1$ and $\psi_{1}(H)=1$ if $|H| \geq 2$. Also, let $\psi_{2}: \overline{\mathfrak{a}^{+}} \rightarrow[0,1]$ be a smooth cutoff function such that $\psi_{2}(H)=0$ if $\omega(H) \leq 1$ and $\psi_{2}(H)=1$ if $\omega(H) \geq 2$. The kernel $K$ in (4.2) can be written as $K=K_{1}+K_{2}+K_{3}$, where

$$
\begin{gathered}
K_{1}(\exp H)=\left(1-\psi_{1}(H)\right) K(\exp H), \\
K_{2}(\exp H)=\psi_{1}(H)\left(1-\psi_{2}(H)\right) K(\exp H),
\end{gathered}
$$

and

$$
K_{3}(\exp H)=\psi_{1}(H) \psi_{2}(H) K(\exp H)
$$

for any $H \in \overline{\mathfrak{a}^{+}}$. The kernels $K_{1}, K_{2}$ and $K_{3}$ are of course $\mathbb{K}$-bi-invariant kernels on $\mathbb{G}$. It follows easily from [1, Corollary 17 ] that the kernel $K_{1}$ defines a bounded operator on $L^{p}(\mathbb{X})$, i.e.,

$$
\left\|\left|\left\|K_{1} \mid\right\|_{L^{p}(\mathbb{X})} \leq C_{p} .\right.\right.
$$


It remains to prove similar estimates on the norms of the operators defined by the kernels $K_{2}$ and $K_{3}$.

Lemma 4.2. One has

$$
\left\||| K_{2} \mid\right\|_{L^{p}(\mathbb{X})} \leq C_{p} .
$$

Proof of Lemma 4.2. This proof is based on the main idea in Anker's paper [1]. By Proposition 2.1] it suffices to prove that

$$
\int_{\mathbb{G}}\left|K_{2}(g)\right| e^{-2 \rho\left(g^{+}\right) / p^{\prime}} d g \leq C_{p} .
$$

As in [1, for any $r \geq 0$ let

$$
V_{r}=\{H \in \mathfrak{a}:(w \cdot \rho)(H)<|\rho| r \text { for any } w \in W\}
$$

and

$$
U_{r}=\mathbb{K}\left(\exp V_{r}\right) \mathbb{K}
$$

We will prove that for any integer $r \geq 0$ one has

$$
\int_{U_{r+1} \backslash U_{r}}\left|K_{2}(g)\right| d g \leq C_{p} e^{2|\rho| r / p^{\prime}}(1+r)^{-3 / 2} .
$$

Notice that $e^{-2 \rho\left(g^{+}\right) / p^{\prime}} \approx e^{-2|\rho| r / p^{\prime}}$ if $g \in U_{r+1} \backslash U_{r}$. Thus (4.4) follows from (4.5) by summation over $r$.

We need the following estimate on the measure of the set $\left(U_{r+1} \backslash U_{r}\right) \cap\left(\operatorname{supp} K_{2}\right)$ :

$$
\left|\left(U_{r+1} \backslash U_{r}\right) \cap \operatorname{supp} K_{2}\right| \leq C(1+r)^{\ell-2} e^{2|\rho| r} .
$$

Notice that the power of $(1+r)$ in (4.6) is $\ell-2$ rather than $\ell-1$ (compare with the estimate in 1, Lemma 6]). This is because of the "small" support of the kernel $K_{2}$, and this gain is essential for the proof of the lemma. To prove (4.6) notice that for any $H \in\left(V_{r+1} \backslash V_{r}\right) \cap \overline{\mathfrak{a}^{+}}$one has

$$
\delta(H) \leq e^{2 \rho(H)} \leq C e^{2|\rho| r},
$$

where $\delta(H)$ is the density measure defined in (2.1). In addition, one can show easily that the measure in $\mathfrak{a}$ of the set $\left(V_{r+1} \backslash V_{r}\right) \cap \overline{\mathfrak{a}^{+}} \cap\left(\operatorname{supp}\left(1-\psi_{2}\right)\right)$ is dominated by $C(1+r)^{\ell-2}$. The estimate (4.6) follows.

By Hölder's inequality and (4.6), one has

$$
\int_{U_{r+1} \backslash U_{r}}\left|K_{2}(g)\right| d g \leq C(1+r)^{(\ell-2) / 2} e^{|\rho| r}|| K_{2} \|_{L^{2}\left(U_{r+1} \backslash U_{r}\right)}
$$

for any integer $r \geq 0$. Thus it suffices to prove that

$$
\|\left. K_{2}\right|_{L^{2}\left(U_{r+1} \backslash U_{r}\right)} \leq C_{p} e^{-\left|\rho_{p}\right| r}(1+r)^{-(\ell+1) / 2} .
$$

Let $\mathcal{H}$ denote the Fourier transform on the symmetric space $\mathbb{X}$ defined in (2.3). It is well known that $\mathcal{H}$ extends to an isomorphism between $\mathbf{S}(\mathbb{G} / / \mathbb{K})$ (the $L^{2}$ Schwartz space of $\mathbb{K}$-bi-invariant functions on $\mathbb{G}$ ) and $\mathbf{S}\left(\mathfrak{a}^{*}\right)^{W}$ (the subspace of $W$-invariant functions in the Schwartz space $\mathbf{S}\left(\mathfrak{a}^{*}\right)$ ). See [1, Section 1] for the definition of $\mathbf{S}(\mathbb{G} / / \mathbb{K})$ and references. For any $f \in C_{c}^{\infty}(\mathbb{G} / / \mathbb{K})$, let $\mathcal{A}(f)$ denote the Abel transform

$$
\mathcal{A}(f)(H)=e^{\rho(H)} \int_{\mathbb{N}} f((\exp H) n) d n .
$$


The Abel transform extends to an isomorphism between $\mathbf{S}\left(\mathbb{G} / / \mathbb{K}\right.$ ) and $\mathbf{S}(\mathfrak{a})^{W}$ (see [1, Proposition 3]). Finally, let $\mathcal{F}$ denote the Euclidean Fourier transform

$$
\mathcal{F}(g)(\lambda)=\int_{\mathfrak{a}} g(H) e^{-i \lambda(H)} d H
$$

which is an isomorphism between $\mathbf{S}(\mathfrak{a})^{W}$ and $\mathbf{S}\left(\mathfrak{a}^{*}\right)^{W}$. In addition, for any $f \in$ $\mathbf{S}(\mathbb{G} / / \mathbb{K})$ one has

$$
\mathcal{H}(f)=\mathcal{F}(\mathcal{A}(f)) \text {. }
$$

Let $L=\mathcal{F}^{-1}(m)=\mathcal{A}(K)$. Assume that $r \geq 2$ in (4.7) (only simple modifications are needed if $r=0$ or $r=1$ ) and let $\Omega_{r}: \mathfrak{a} \rightarrow[0,1]$ be the $W$-invariant smooth cutoff functions defined in [1 Section 2]. These functions are supported in the complement of $V_{r-1}$ and are equal to 1 in the complement of $V_{r}$. The key observation in [1. Section 2] is that

$$
K(\exp H)=\mathcal{A}^{-1}\left(L \cdot \Omega_{r}\right)(H)
$$

for any $H$ outside $U_{r}$. This is a simple consequence of a support property of the Abel transform (see [1, Proposition 4]). Thus, by the Plancherel theorem, one has

$$
\begin{aligned}
\left\|K_{2}\right\|_{L^{2}\left(U_{r+1} \backslash U_{r}\right)} & \leq\|K\|_{L^{2}\left(\mathbb{G} \backslash U_{r}\right)} \leq C\left\|\mathcal{A}^{-1}\left(L \cdot \Omega_{r}\right)\right\|_{L^{2}(\mathbb{G})} \\
& \leq C\left\|\mathcal{F}\left(L \cdot \Omega_{r}\right)\right\|_{L^{2}\left(\mathfrak{a}^{*},|\mathbf{c}(\lambda)|^{-2} d \lambda\right)} .
\end{aligned}
$$

One also has the estimate

$$
|\mathbf{c}(\lambda)|^{-2} \leq C\left(1+|\lambda|^{2}\right)^{2 b}
$$

for any $\lambda \in \mathfrak{a}^{*}$, where $b$ is a fixed positive integer. By the Euclidean Plancherel theorem

$$
\begin{aligned}
\left\|\mathcal{F}\left(L \cdot \Omega_{r}\right)\right\|_{L^{2}\left(\mathfrak{a}^{*},|\mathbf{c}(\lambda)|^{-2} d \lambda\right)} & \leq C\left\|\mathcal{F}\left(L \cdot \Omega_{r}\right)(\lambda)\left(1+|\lambda|^{2}\right)^{b}\right\|_{L^{2}\left(\mathfrak{a}^{*}\right)} \\
& \leq C \sum_{j=0}^{b}\left\|\Delta_{\mathfrak{a}}^{j}\left(L \cdot \Omega_{r}\right)\right\|_{L^{2}(\mathfrak{a})},
\end{aligned}
$$

where $\Delta_{\mathfrak{a}}$ is the Laplace-Beltrami operator on $\mathfrak{a}$. Thus it suffices to prove that for any integer $j \in\{0,1, \ldots, b\}$ one has

$$
\left\|\Delta_{\mathfrak{a}}^{j}\left(L \cdot \Omega_{r}\right)\right\|_{L^{2}(\mathfrak{a})} \leq C_{p} e^{-\left|\rho_{p}\right| r}(1+r)^{-(\ell+1) / 2} .
$$

Recall that $L$ is the inverse Euclidean Fourier transform of the multiplier $m$; therefore,

$$
L(H)=C \int_{\mathfrak{a}^{*}} m(\lambda) e^{i \lambda(H)} d \lambda .
$$

Assume that $H \in \overline{\mathfrak{a}^{+}}$and $\rho(H) \geq 1$. Since the multiplier $m$ is holomorphic, one can shift the integration in (4.9) to the space $i(1-\varepsilon) \rho_{p}+\mathfrak{a}^{*}$, where $\varepsilon \rho(H)=1$. One has

$$
L(H)=C_{p} e^{-\rho_{p}(H)} \int_{\mathfrak{a}^{*}} m\left(\lambda+i(1-\varepsilon) \rho_{p}\right) e^{i \lambda(H)} d \lambda .
$$


We integrate by parts $N$ times in $\lambda$ and use (4.1). Notice also that if $H \in \overline{{ }^{\top} \mathfrak{a}}$, then $\rho(H) \approx|H|$. Therefore one has

$$
\begin{aligned}
|L(H)| & \leq C_{p} e^{-\rho_{p}(H)}|H|^{-2 N} \int_{\mathfrak{a}^{*}}\left|\Delta_{\mathfrak{a}^{*}}^{N} m\left(\lambda+i(1-\varepsilon) \rho_{p}\right)\right| d \lambda \\
& \leq C_{p} e^{-\rho_{p}(H)}|H|^{-2 N} \int_{\mathfrak{a}^{*}}(\varepsilon+|\lambda|)^{-2 N} d \lambda \\
& \leq C_{p} e^{-\rho_{p}(H)} \rho(H)^{-\ell}
\end{aligned}
$$

if $2 N \geq \ell+1$ and $H \in \overline{{ }^{+} \mathfrak{a}} \cap\left(\mathfrak{a} \backslash V_{r-1}\right)$. The kernel $L$ is $W$-invariant and the measure (in $\mathfrak{a}$ ) of the set $V_{r^{\prime}} \backslash V_{r^{\prime}-1}$ is dominated by $\left(1+r^{\prime}\right)^{\ell-1}$ for any $r^{\prime} \geq 1$. The estimate (4.8) follows for $j=0$. One can easily repeat the argument for any $j \leq b$ and Lemma 4.2 follows.

It remains to prove the following estimate:

Lemma 4.3. One has

$$
\left.||\left|* K_{3}\right|\right|_{L^{p}(\mathbb{X})} \leq C_{p} .
$$

Proof of Lemma 4.3. We will use the Harish-Chandra expansion of the spherical functions $\varphi_{\lambda}(H)$. Let

$$
\mathfrak{a}_{+}^{*}=\left\{\lambda \in \mathfrak{a}^{*}: H_{\lambda} \in \mathfrak{a}^{+}\right\}
$$

and let

$$
B=\left\{\lambda \in \mathfrak{a}^{*}: \alpha\left(H_{\lambda}\right)>-\varepsilon_{0} \text { for any } \alpha \in \Sigma^{+}\right\}
$$

be an open neighborhood of $\overline{\mathfrak{a}_{+}^{*}}$. The constant $\varepsilon_{0}$ will be chosen small and strictly positive. Let $Q$ be the positive lattice generated by the simple roots $\alpha \in \Sigma^{+}$. If $H \in \mathfrak{a}^{+}$and $\lambda \in \mathfrak{a}^{*}$, then one has the absolutely converging expansion

$$
|\mathbf{c}(\lambda)|^{-2} \varphi_{\lambda}(H)=e^{-\rho(H)} \sum_{q \in 2 Q} e^{-q(H)} \sum_{w \in W} \mathbf{c}(-w \cdot \lambda)^{-1} \Gamma_{q}(w \cdot \lambda) e^{i(w \cdot \lambda)(H)} .
$$

The coefficient $\Gamma_{0}$ is equal to 1 ; the other coefficients $\Gamma_{q}$ are rational functions in $\lambda$ and extend to holomorphic functions in the tube $\mathfrak{a}^{*}+i B$ if the constant $\varepsilon_{0}$ is chosen small enough. Moreover, there exist constants $C$ and $d$ such that

$$
\left|\Gamma_{q}(\lambda)\right| \leq C(1+|q|)^{d}
$$

for any $\lambda \in \mathfrak{a}^{*}+i B$ and $q \in Q$. We substitute the expansion (4.11) into the inversion formula (4.2) and notice that the integrand is $W$-invariant. One has

$$
K_{3}(\exp H)=C \psi_{1}(H) \psi_{2}(H) e^{-\rho(H)} \sum_{q \in Q} e^{-q(H)} \int_{\mathfrak{a}^{*}} m(\lambda) \mathbf{c}(-\lambda)^{-1} \Gamma_{q}(\lambda) e^{i \lambda(H)} d \lambda .
$$

The main term in the sum above corresponds to $q=0$. We use the Herz majorizing principle to estimate the $L^{p}$ norms of the operators induced by the error terms in (4.13). For any $q \in Q$ let

$$
K_{3}^{q}(\exp H)=C \psi_{1}(H) \psi_{2}(H) e^{-\rho(H)} e^{-q(H)} \int_{\mathfrak{a}^{*}} m(\lambda) \mathbf{c}(-\lambda)^{-1} \Gamma_{q}(\lambda) e^{i \lambda(H)} d \lambda
$$

In general (i.e., if $\ell \geq 2$ ) the functions $\Gamma_{q}$ do not satisfy favorable symbol-type estimates on $\mathfrak{a}^{*}$ except for the uniform inequalities (4.12). However, one can use the fact that they are holomorphic functions in the tube $\mathfrak{a}^{*}+i B$ to estimate the absolute value of $K_{3}^{q}(\exp H)$. This idea was used in a recent work of Anker and Ji 
[3]. See also [2] for similar estimates. Let $\Sigma^{++}$denote the set of indivisible roots $\alpha \in \Sigma^{+}$and let

$$
P(\lambda)=\prod_{\alpha \in \Sigma^{++}}(1-i\langle\alpha, \lambda\rangle)^{k_{\alpha}},
$$

where $k_{\alpha}$ are certain large integers. The function $\mathbf{c}$ can be computed explicitly (see, for example, [2, Section 2]). This explicit formula shows that the function $\lambda \rightarrow \mathbf{c}(-\lambda)^{-1}$ is holomorphic in the tube $\mathfrak{a}^{*}+i B$ if $\varepsilon_{0}$ is small enough. In addition, there are positive constants $k_{\alpha}{ }^{\prime}$ such that

$$
\left|\mathbf{c}(-\lambda)^{-1}\right| \leq C \prod_{\alpha \in \Sigma^{++}}|1-i\langle\alpha, \lambda\rangle|^{k_{\alpha}}
$$

for any $\lambda \in \mathfrak{a}^{*}+i B$. Thus one can fix the constants $k_{\alpha}$ in (4.15) so that the function c $(-\lambda)^{-1} / P(\lambda)$ belongs to the space $H^{2}\left(T_{B}\right)$ - see [19, Chapter III] for the theory of $H^{p}$ spaces on tubes. By (4.12) the function $\lambda \rightarrow \Gamma_{q}(\lambda) \mathbf{c}(-\lambda)^{-1} / P(\lambda)$ belongs to the space $H^{2}\left(T_{B}\right)$ and

$$
\int_{\mathfrak{a}^{*}}\left|\Gamma_{q}(\eta+i \xi) \mathbf{c}(-\eta-i \xi)^{-1} / P(\eta+i \xi)\right|^{2} d \eta \leq C(1+|q|)^{2 d}
$$

for any $\xi \in B$. One has

$$
\begin{aligned}
K_{3}^{q}(\exp H) & =C \psi_{1}(H) \psi_{2}(H) e^{-\rho(H)} e^{-q(H)} \mathcal{F}^{-1}\left(\lambda \rightarrow m(\lambda) \mathbf{c}(-\lambda)^{-1} \Gamma_{q}(\lambda)\right) \\
& =C \psi(H) e^{-\rho(H)} e^{-q(H)} \mathcal{F}^{-1}(m \cdot P) * \mathcal{F}^{-1}\left(f_{q}\right)(H),
\end{aligned}
$$

where $\psi=\psi_{1} \psi_{2}$ and $f_{q}(\lambda)=\Gamma_{q}(\lambda) \mathbf{c}(-\lambda)^{-1} / P(\lambda)$. By 19, Theorem 3.1, Section III], the function $\mathcal{F}^{-1}\left(f_{q}\right)$ is supported in the cone $-\overline{{ }^{+} \mathfrak{a}}$ and satisfies

$$
\int_{-\overline{+}_{\mathfrak{a}}}\left|\mathcal{F}^{-1}\left(f_{q}\right)(H)\right|^{2} d H \leq C(1+|q|)^{2 d} .
$$

On the other hand, $\mathcal{F}^{-1}(m \cdot P)$ is a certain derivative (in $H$ ) of the function $L=$ $\mathcal{F}^{-1}(m)$. An argument similar to the one at the end of Lemma 4.2 (see (4.10) shows that

$$
\left|\mathcal{F}^{-1}(m \cdot P)(H)\right| \leq C_{p} e^{-\rho_{p}(H)}(1+\rho(H))^{-\ell}
$$

for any $H \in \overline{{ }^{+} \mathfrak{a}}$ with the property that $|H| \geq 1$. Combining the last three equations and Hölder's inequality one has

$$
\begin{aligned}
& \left|K_{3}^{q}(\exp H)\right| \\
& \quad \leq C_{p} \psi(H) e^{-\rho(H)} e^{-q(H)} \int_{\mathfrak{a}}\left|\mathcal{F}^{-1}(m \cdot P)\left(H-H^{\prime}\right)\right|\left|\mathcal{F}^{-1}\left(f_{q}\right)\left(H^{\prime}\right)\right| d H^{\prime} \\
& \quad \leq C_{p}(1+|q|)^{d} \psi(H) e^{-\rho(H)} e^{-q(H)}\left(\int_{\overline{+_{\mathfrak{a}}}}\left|\mathcal{F}^{-1}(m \cdot P)\left(H+H^{\prime}\right)\right|^{2} d H^{\prime}\right)^{1 / 2} \\
& \quad \leq C_{p}(1+|q|)^{d} \psi(H) e^{-2 \rho(H) / p} e^{-|q| \omega(H)}(1+\rho(H))^{-\ell}
\end{aligned}
$$

for any $H \in \overline{\mathfrak{a}^{+}}$. The Herz majorizing principle applies if $|q| \geq 1$. Recall that the support of the cutoff function $\psi$ is included in the set $\left\{H \in \overline{\mathfrak{a}^{+}}: \omega(H) \geq 1\right\}$. By 
(2.7) and Lemma 3.5 one has

$$
\begin{aligned}
\|\| * K_{3}^{q}||_{L^{p}(\mathbb{X})} & \leq C_{p} \int_{\overline{\mathfrak{a}^{+}}}\left|K_{3}^{q}(\exp H)\right| \delta(H) e^{-2 \rho(H) / p^{\prime}} d H \\
& \leq C_{p}(1+|q|)^{d} e^{-(|q|-1)} \int_{\overline{\mathfrak{a}^{+}}}(1+|H|)^{-\ell} e^{-\omega(H)} d H \\
& \leq C_{p}(1+|q|)^{d} e^{-|q|} .
\end{aligned}
$$

It remains to deal with the kernel $K_{3}^{0}$. We need one more reduction before we can apply the results in the previous section. For any $H \in \overline{\mathfrak{a}^{+}}$let

$$
\widetilde{K_{3}^{0}}(\exp H)=C \psi_{1}(H) e^{-\rho(H)} \int_{\mathfrak{a}^{*}} m(\lambda) \mathbf{c}(-\lambda)^{-1} e^{i \lambda(H)} d \lambda
$$

(compare with (4.14) ) and extend $\widetilde{K_{3}^{0}}$ to a $\mathbb{K}$-bi-invariant function on $\mathbb{G}$. Notice that the function $H \rightarrow\left(K_{3}^{0}(\exp H)-\widetilde{K_{3}^{0}}(\exp H)\right)$ is supported in the set $\{H \in$ $\left.\overline{\mathfrak{a}^{+}}: \omega(H) \leq 2\right\}$ and satisfies an estimate similar to (4.16) for $q=0$. By (2.7) and Lemma 3.5 one has

$$
\left\|\left|*\left(K_{3}^{0}-\widetilde{K_{3}^{0}}\right)\right|\right\|_{L^{p}(\mathbb{X})} \leq C_{p} .
$$

It remains to prove a similar inequality for the kernel $\widetilde{K_{3}^{0}}$. This will be a consequence of Corollary 3.2 For any $H \in \overline{\mathfrak{a}^{+}}$let

$$
\phi(H)=e^{2 \rho(H) / p} K_{3}^{0}(\exp H)=C \psi_{1}(H) e^{\rho_{p}(H)} \int_{\mathfrak{a}^{*}} m(\lambda) \mathbf{c}(-\lambda)^{-1} e^{i \lambda(H)} d \lambda
$$

and $\phi(H)=0$ if $H \notin \overline{\mathfrak{a}^{+}}$. We have to prove that $\phi$ satisfies conditions (3.3) and (3.4) in the previous section. Notice that for any $H \in \overline{\mathfrak{a}^{+}}$one has

$$
\begin{aligned}
& \phi(H)=C \psi_{1}(H) e^{\rho_{p}(H)} \mathcal{F}^{-1}(m \cdot P) * \mathcal{F}^{-1}\left(f_{0}\right)(H) \\
& \quad=C \psi_{1}(H) \int_{\mathfrak{a}}\left(e^{\rho_{p}\left(H-H^{\prime}\right)} \mathcal{F}^{-1}(m \cdot P)\left(H-H^{\prime}\right)\right)\left(e^{\rho_{p}\left(H^{\prime}\right)} \mathcal{F}^{-1}\left(f_{0}\right)\left(H^{\prime}\right)\right) d H^{\prime} .
\end{aligned}
$$

Recall also that $\mathcal{F}^{-1}\left(f_{0}\right)$ is an $L^{2}$ functions supported in the set $-\overline{{ }^{+} \mathfrak{a}}$. Therefore the estimate (3.3) is an easy consequence of the following estimate:

$$
\partial_{H}^{j}\left(H \rightarrow e^{\rho_{p}(H)} \mathcal{F}^{-1}(m \cdot P)(H)\right) \leq C_{p}(1+|H|)^{-\ell-j}
$$

for any $H \in \overline{{ }^{+} \mathfrak{a}}$ with the property that $\rho(H) \geq 1$ and for $j=0$ or $j=1$. Let

$$
\widetilde{\phi}(H)=e^{\rho_{p}(H)} \mathcal{F}^{-1}(m \cdot P)(H) .
$$

The proof of (4.18) for $j=0$ is identical to the proof of (4.10) in Lemma 4.2. We will now prove (4.18) with $j=1$ and $H=H_{0} \in \overline{{ }^{+} \mathfrak{a}}$ away from the origin. Let $\varepsilon=\rho\left(H_{0}\right)^{-1}$ and notice that

$$
\widetilde{\phi}(H)=e^{\rho_{p}(H) / \rho\left(H_{0}\right)} \int_{\mathfrak{a}^{*}} m\left(\lambda+i(1-\varepsilon) \rho_{p}\right) P\left(\lambda+i(1-\varepsilon) \rho_{p}\right) e^{i \lambda(H)} d \lambda
$$


by a shift of integration to the space $\mathfrak{a}^{*}+i(1-\varepsilon) \rho_{p}$. Fix $H_{1} \in \mathfrak{a}$ with $\left|H_{1}\right|=1$. One has

$$
\begin{aligned}
\left|\frac{\partial \widetilde{\phi}}{\partial H_{1}}\left(H_{0}\right)\right| & \leq C \rho\left(H_{0}\right)^{-1}\left|\int_{\mathfrak{a}^{*}} m\left(\lambda+i(1-\varepsilon) \rho_{p}\right) P\left(\lambda+i(1-\varepsilon) \rho_{p}\right) e^{i \lambda\left(H_{0}\right)} d \lambda\right| \\
& +C\left|\int_{\mathfrak{a}^{*}} m\left(\lambda+i(1-\varepsilon) \rho_{p}\right) P\left(\lambda+i(1-\varepsilon) \rho_{p}\right) \lambda\left(H_{1}\right) e^{i \lambda\left(H_{0}\right)} d \lambda\right| .
\end{aligned}
$$

Let $C_{1}$ be the degree of the polynomial $P$. The estimate (4.18) for $j=1$ follows by integration by parts as in (4.10). One only needs to notice that, as a consequence of (4.1),

$$
\mid \Delta_{\mathfrak{a}^{*}}^{N}\left(\lambda \rightarrow m\left(\lambda+i(1-\varepsilon) \rho_{p}\right) P\left(\lambda+i(1-\varepsilon) \rho_{p}\right) \mid \leq C_{N}(\varepsilon+|\lambda|)^{-2 N}(1+|\lambda|)^{C_{1}}\right.
$$

and

$$
\begin{aligned}
\left|\Delta_{\mathfrak{a}^{*}}^{N}\left(\lambda \rightarrow m\left(\lambda+i(1-\varepsilon) \rho_{p}\right) P\left(\lambda+i(1-\varepsilon) \rho_{p}\right) \lambda\left(H_{1}\right)\right)\right| & \\
& \leq C_{N}(\varepsilon+|\lambda|)^{1-2 N}(1+|\lambda|)^{C_{1}}
\end{aligned}
$$

for any integer $N \geq 0$, where, as before, $\Delta_{\mathfrak{a}^{*}}$ denotes the Laplace-Beltrami operator on $\mathfrak{a}^{*}$.

The proof of the cancellation condition (3.4) is somewhat delicate, mainly because the support of the function $\phi$ is restricted to the cone $\overline{\mathfrak{a}^{+}}$. We proceed as in 14]. Let $\mu: \mathbb{R} \rightarrow[0,1]$ be a smooth cutoff function supported in the interval $[1, \infty)$ and equal to 1 in the interval $[2, \infty)$. Let $\Sigma^{+++}$be the set of simple roots in $\Sigma^{+}$ and for any $H \in \mathfrak{a}$ let

$$
\Psi(H)=\prod_{\alpha \in \Sigma^{+++}} \mu(\alpha(H)) .
$$

Notice that we can replace the cutoff function $\psi_{1}$ in (4.17) with $\Psi$. Indeed, let

$$
\phi_{1}(H)=C \Psi(H) e^{\rho_{p}(H)} \int_{\mathfrak{a}^{*}} m(\lambda) \mathbf{c}(-\lambda)^{-1} e^{i \lambda(H)} d \lambda
$$

and notice that the function $\phi-\phi_{1}$ is supported in the set $\left\{H \in \overline{\mathfrak{a}^{+}}: \omega(H) \leq 2\right\}$ and satisfies the estimate (3.3) for $j=0$. Thus $\phi-\phi_{1}$ is an $L^{1}$ function. It remains to prove that the function $\phi_{1}$ satisfies the cancellation condition (3.4). Recall that

$$
\left|\phi_{1}(H)\right| \leq C(1+|H|)^{-\ell}
$$

for any $H \in \mathfrak{a}$. Thus the cancellation condition (3.4) is equivalent to

$$
\left|\int_{\mathfrak{a}} \phi_{1}(H) \gamma(\varepsilon H)\right| \leq C
$$

for any $\varepsilon \in(0,1 / 2]$, where $\gamma: \mathfrak{a} \rightarrow[0,1]$ is a smooth cutoff function supported in the ball $\{H \in \mathfrak{a}:|H| \leq 2\}$ and equal to 1 in the ball $\{H \in \mathfrak{a}:|H| \leq 1\}$. Let $T=\mathcal{F}^{-1}(\Psi)$ be a distribution on $\mathfrak{a}^{*}$. By the same argument as in [14, Section 4], it suffices to prove that

$$
\left|T\left(\eta \rightarrow \widetilde{m}\left(\eta-\eta_{0}+i(1-\varepsilon) \rho_{p}\right)\right)\right| \leq C\left(1+\left|\eta_{0}\right|\right)^{C_{1}}
$$

for any $\eta_{0} \in \mathfrak{a}^{*}$ and any $\varepsilon \in(0,1]$, where $C_{1}$ is the same constant as before and $\widetilde{m}(\lambda)=m(\lambda) \mathbf{c}(-\lambda)^{-1}$. For any $\alpha \in \Sigma^{+++}$let $T_{\alpha}=\mathcal{F}^{-1}(H \rightarrow \mu(\alpha(H)))$. One has

$$
T(f)=T_{\alpha_{1}} * \cdots * T_{\alpha_{\ell}}(f)
$$

for any Schwartz function $f: \mathfrak{a} \rightarrow \mathbb{C}$, where $\alpha_{1}, \ldots, \alpha_{\ell}$ are the positive simple roots. The estimate (4.20) will be a consequence of the following lemma: 
Lemma 4.4. Assume that $D_{1}$ is an open set in the Euclidean space $\mathbb{R}^{n}, u \in \mathbb{R}^{n}$ is a fixed vector with $|u|=1$ and $D_{2}$ is an open set with the property that $D_{2}+t u \subset D_{1}$ for any $t \in\left[0, \delta_{0}\right], \delta_{0}>0$. Let $T_{u}$ be the inverse Fourier transform of the function $v \rightarrow \mu(\langle u, v\rangle)$ (in the sense of distributions). Assume that $f$ is a holomorphic function in the tube $\mathbb{R}^{n}+i D_{1}$ with the property that

$$
|f(x+i y)| \leq A(1+|x|)^{\delta_{1}}
$$

for any $x \in \mathbb{R}^{n}$ and $y \in D_{1}$. In addition, assume that the function $x \rightarrow f(x+i y)$ is a Schwartz function on $\mathbb{R}^{n}$ for any $y \in D_{1}$. Then the function

$$
f * T_{u}(x+i y)=T_{u}(v \rightarrow f(x-v+i y))
$$

has the property that

$$
\left|f * T_{u}(x+i y)\right| \leq C_{\delta_{0}} \cdot A(1+|x|)^{\delta_{1}}
$$

for any $x \in \mathbb{R}^{n}$ and $y \in D_{2}$. In addition, the function $x \rightarrow f * T_{u}(x+i y)$ is a Schwartz function on $\mathbb{R}^{n}$ for any $y \in D_{2}$. The constant $C_{\delta_{0}}$ depends only on $\delta_{0}$ and the cutoff function $\mu$.

The estimate (4.20) follows from this lemma by a simple inductive argument. One starts with $f(\lambda)=\widetilde{m}(-\lambda)$ and $D_{1}=-\operatorname{co}\left(W \cdot \rho_{p}\right)$ and applies Lemma 4.4 to the vectors $u=H_{\alpha_{1}}, \ldots, H_{\alpha_{\ell}}$. One only needs to check that there exists a small constant $\delta_{0}=\delta_{0}(p)$ with the property that

$$
(1-\varepsilon) \rho_{p}-\left(t_{1} \alpha_{1}+\ldots+t_{\ell} \alpha_{\ell}\right) \in \operatorname{co}\left(W \cdot \rho_{p}\right)
$$

for any $t_{1}, \ldots, t_{\ell} \in\left[0, \delta_{0}\right]$. Recall that $\varepsilon \in(0,1 / 2]$. We need the following wellknown fact about convex hulls (see [11, Chapter IV]): if $H \in \overline{\mathfrak{a}^{+}}$and $C(H)$ is the closure of the orbit $\{w \cdot H: w \in W\}$, then

$$
C(H) \cap \overline{\mathfrak{a}^{+}}=\left(H-\overline{{ }^{+} \mathfrak{a}}\right) \cap \overline{\mathfrak{a}^{+}} .
$$

We can pass this to the space $\mathfrak{a}^{*}$ and apply it to the vector $\rho$. One has

$$
C(\rho) \cap \overline{\mathfrak{a}_{+}^{*}}=\left(\rho-\overline{+\mathfrak{a}^{*}}\right) \cap \overline{\mathfrak{a}_{+}^{*}}
$$

where $C(\rho)$ is the closure of the set $\operatorname{co}(W \cdot \rho)$ and, as before, $\mathfrak{a}_{+}^{*}$ and ${ }_{+} \mathfrak{a}^{*}$ are the cones corresponding to $\mathfrak{a}^{+}$and ${ }^{+} \mathfrak{a}$, respectively. Notice also that $\alpha_{1}, \ldots, \alpha_{\ell} \in \overline{+\mathfrak{a}^{*}}$. Thus one has

$$
\rho-\left(t_{1} \alpha_{1}+\ldots+t_{\ell} \alpha_{\ell}\right) \in C(\rho)
$$

for any $t_{1}, \ldots, t_{\ell} \in[0, c]$ and (4.21) follows.

Proof of Lemma 4.4. This is a consequence of the one-dimensional estimate proved in [14, Section 4]. Assume that $u=(1,0, \ldots, 0)$. The distribution $T_{u}$ is the inverse Fourier transform of the function $v \rightarrow \mu\left(v_{1}\right)$. The same argument as in [14] Section 4] shows that

$$
T_{u}(f)=\int_{\mathbb{R}} \frac{\nu(t)\left(f(t, 0, \ldots, 0)-f(0) e^{-t^{2}}\right)}{-i t} d t+C f(0)
$$

for any Schwartz function $f$, where $\nu$ is the inverse Fourier transform of the function $\mu^{\prime}$. Thus

$$
f * T_{u}(x+i y)=\int_{\mathbb{R}} \frac{\nu(t)\left(f(x-t u+i y)-f(x+i y) e^{-t^{2}}\right)}{-i t} d t+C f(x+i y)
$$


where $f$ is the function in Lemma 4.4 and $y \in D_{2}$. The function $\nu$, the inverse Fourier transform of the smooth compactly supported function $\mu^{\prime}$, extends to a holomorphic function in the plane. We shift the integration in (4.22) to the line $-i \delta_{0}+\mathbb{R}$, where $\delta_{0}$ is the constant in Lemma 4.4. Notice also that the function $t \rightarrow \nu\left(t-i \delta_{0}\right)$ is a Schwartz function on $\mathbb{R}$ and the lemma follows.

\section{REFERENCES}

[1] J.-Ph. Anker, $L^{p}$ Fourier multipliers on Riemannian symmetric spaces of the noncompact type, Ann. of Math. 132 (1990), 597-628. MR 92e:43006

[2] J.-Ph. Anker and L. Ji, Heat kernel and Green function estimates on noncompact symmetric spaces, Geom. Funct. Anal. 9 (1999), 1035-1091. MR 2001b:58038

[3] J.-Ph. Anker and L. Ji, Heat kernel and Green function estimates on noncompact symmetric spaces II, Preprint (1999).

[4] J.-Ph. Anker and N. Lohoué, Multiplicateurs sur certains espaces symétriques, Amer. J. Math. 108 (1986), 1303-1354. MR 88c:43008

[5] J.-L. Clerc and E. M. Stein, $L^{p}$-multipliers for noncompact symmetric spaces, Proc. Nat. Acad. Sci. U.S.A. 71 (1974), 3911-3912. MR 51:3803

[6] R. Coifman and G. Weiss, Transference Methods in Analysis, CBMS Regional Conference Series in Mathematics, No. 31, Amer. Math. Soc., Providence, RI (1976). MR 58:2019

[7] M. Cowling, The Kunze-Stein phenomenon, Ann. Math. 107 (1978), 209-234. MR 58:22398

[8] M. Cowling, S. Giulini and S. Meda, $L^{p}-L^{q}$ estimates for functions of the Laplace-Beltrami operator on noncompact symmetric spaces. I, Duke Math. J. 72 (1993), 109-150. MR 95b:22031

[9] S. Giulini, G. Mauceri and S. Meda, $L^{p}$ multipliers on noncompact symmetric spaces, J. reine angew. Math. 482 (1997), 151-175. MR 98g:43006

[10] Harish-Chandra, Spherical functions on a semisimple Lie group. I, Amer. J. Math. 80 (1958), 241-310. MR 20:925

[11] S. Helgason, Groups and Geometric Analysis; Integral Geometry, Invariant Differential Operators and Spherical Functions, Academic Press, New York (1984). MR 86c:22017

[12] S. Helgason, Geometric Analysis on Symmetric Spaces, Amer. Math. Soc., Providence, RI (1994). MR 96h:43009

[13] C. Herz, Sur le phénomène de Kunze-Stein, C. R. Acad. Sci. Paris, Série A 271 (1970), 491-493. MR 43:6741

[14] A. D. Ionescu, Singular integrals on symmetric spaces of real rank one, Duke Math. J. 114 (2002), 101-122. MR 2003c:43008

[15] R. A. Kunze and E. M. Stein, Uniformly bounded representations and harmonic analysis of the $2 \times 2$ unimodular group, Amer. J. Math. 82 (1960), 1-62. MR 29:1287

[16] L.-A. Lindahl, Fatou's theorem for symmetric spaces, Ark. Mat. 10 (1972), 33-47. MR 52:3892

[17] N. Lohoué and T. Rychener, Some function spaces on symmetric spaces related to convolution operators, J. Funct. Anal. 55 (1984), 200-219. MR 85d:22024

[18] R. J. Stanton and P. A. Tomas, Expansions for spherical functions on noncompact symmetric spaces, Acta Math. 140 (1978), 251-271. MR 58:23365

[19] E. M. Stein, Introduction to Fourier Analysis on Euclidean Spaces, Princeton Univ. Press (1971). MR 46:4102

[20] E. M. Stein, Harmonic Analysis, Princeton Univ. Press (1993). MR 95c:42002

[21] J. O. Strömberg, Weak type $L^{1}$ estimates for maximal functions on noncompact symmetric spaces, Ann. Math. 114 (1981), 115-126. MR 82k:43010

[22] M. E. Taylor, $L^{p}$ estimates on functions of the Laplace operator, Duke Math. J. 58 (1989), 773-793. MR 91d:58253

Massachusetts Institute of Technology, Cambridge, Massachusetts 02139

E-mail address: aionescu@math.mit.edu

Current address: University of Wisconsin - Madison, Madison, Wisconsin 53706

E-mail address: ionescu@math.wisc.edu 